\title{
Perfil da dermatite atópica canina em Salvador - Brasil
}

\author{
Monique Graziele Oliveira Santos ${ }^{1 *} \&$ Monica Mattos dos Santos ${ }^{2}$ \\ ${ }^{I}$ Médica Veterinária Autônoma, pós-graduanda em dermatologia veterinária pela Equalis, Universidade Federal da Bahia, \\ Salvador, Bahia, Brasil. \\ ${ }^{2}$ Prof. Dra. Curso de Medicina Veterinária - Universidade Federal da Bahia, Salvador - BA, Brasil \\ *Autor para correspondência: E-mail: mgos.vet00@yahoo.com.br
}

RESUMO. A dermatite atópica canina apresenta-se como uma reação de hipersensibilidade do tipo I, mediada pela imunoglobulina E (IgE) e por defeitos na barreira epidérmica e na codificação das proteínas de adesão. Por possuir um envolvimento hereditário em sua patogenia, o histórico familiar, assim como a predisposição racial, são importantes fatores de risco para o desenvolvimento desta afecção. Apesar de ser descrita em outras regiões do Brasil como a segunda afecção alérgica mais comum, na Bahia são escassas as informações sobre a frequência desta dermatopatia. Desta forma, objetiva-se determinar a frequência de dermatite atópica canina no Hospital Universitário Veterinário da cidade Salvador, Bahia. Para tanto, foram analisadas as 3375 fichas do Hospital Universitário Veterinário, que correspondiam a todos os novos atendimentos no período de janeiro a dezembro de 2012. Dentro deste universo foram separadas e catalogadas as fichas clínicas que apresentavam queixas dermatológicas. Desta forma, os resultados revelaram que $21,5 \%$ das fichas eram referentes a afecções do sistema tegumentar, e, dentre estas, $17,9 \%$ eram condizentes com enfermidades alérgicas. Quase 30\% destas reações correspondiam a atopia, confirmando-a como a segunda afecção de hipersensibilidade mais frequente no Hospital Veterinário Universitário. Tal estudo demonstra que as afecções dermatológicas estão entre as principais queixas na rotina clínica e reitera a necessidade de um traçado nacional do perfil epidemiológico das afecções dermatológicas.

Palavras chave: Alergia, atopia, cães, dermatologia, hospital, veterinário

\section{Profile of canine atopic dermatitis in Salvador - Brazil}

\begin{abstract}
The canine atopic dermatitis presented as a Type I hypersensitivity reaction mediated by immunoglobulin E (IgE) and defects in the epidermal barrier and the coding of adhesion proteins. By owning a hereditary involvement in its pathogenesis, family history, as well as racial predisposition, they are important risk factors for developing this disease. Despite being described in other regions of Brazil as the second most common allergic disease in Bahia is little information about the frequency of this skin disease. Thus, the objective is to determine the frequency of canine atopic dermatitis at the Veterinary Teaching Hospital of the city Salvador, Bahia. Therefore, we analyzed the 3375 records of the Veterinary Teaching Hospital, which accounted for all new appointments in the period January to December 2012. Within this universe were separated and cataloged the medical records that had skin complaints. Thus, the results showed that $21.5 \%$ of the chips were related to the integumentary system diseases, and among these, $17.9 \%$ were consistent with allergic diseases. Almost 30\% of these reactions corresponded to atopy, confirming it as the second most frequent hypersensitivity condition at University Veterinary Hospital. This study demonstrates that skin diseases are among the main complaints in the clinical routine and reiterates the need for a national tracing epidemiological profile of dermatologic disorders.
\end{abstract}

Keywords: allergy, atopy, dermatology, dogs, hospital, veterinarian 


\section{Introdução}

A dermatite atópica canina é o segundo distúrbio alérgico mais comum, sendo menos frequente apenas que a DAPE (Zanon et al., 2008). Por possuir um envolvimento hereditário em sua patogenia, o histórico familiar, assim como a predisposição racial, são importantes fatores de risco para o desenvolvimento desta afecção (Marsella \& Samuelson, 2009). A prevalência de atopia na população canina a nível mundial é de aproximadamente 10 a $15 \%$, sendo os cães intradomiciliares os mais predispostos a esta afecção (Favrot et al., 2010), percentuais semelhantes também são observados no Brasil (Bianchi et al., $\underline{2008}$, Souza et al., 2009). São poucos os trabalhos que traçam o perfil epidemiológico das afecções dermatológicas nas diferentes regiões do Brasil, sendo registrados até $\mathrm{o}$ momento maior prevalência das dermatopatias bacterianas (20,5 $30,35 \%)$ seguidas pelas parasitárias (15,56 $19,3 \%)$, fúngicas $(4-14,79 \%)$ e imunológicas $(10,51-37,8 \%)$ (Cardoso et al., 2011, Souza et al., 2009). Assim, a escassez de dados sobre a frequência de dermatopatias na Bahia, especialmente atopia, reflete o pequeno número de pesquisas realizadas sobre o assunto. Desta forma, o objetivo desse trabalho é determinar a frequência de dermatite atópica canina no Hospital Universitário Veterinário da cidade de Salvador, Bahia.

\section{Material e Métodos}

Foram analisados 3.375 prontuários clínicos iniciados entre janeiro de 2012 e dezembro de 2012 no Hospital de Medicina Veterinária Professor Renato de Medeiros Neto, da Universidade Federal da Bahia (HOSPMEV). Os prontuários eram preenchidos pelo Médico Veterinário responsável durante o exame clínico e informações adicionais eram inseridas no retorno dos pacientes à consulta.

Os atendimentos classificados como dermatológicos foram analisados quanto ao sexo, idade, raça e afecção. As patologias do tecido tegumentar foram subdividas em alterações dermatológicas de origem endócrina, neoplásica, psicogênica, fúngica, bacteriana, parasitária e alérgica. Alguns dos pacientes com afecções dermatológicas ainda estão sem diagnóstico, seja porque os resultados dos testes laboratoriais não ficaram disponíveis para adequada classificação ou porque é um caso que demanda uma investigação mais profunda e cuidadosa, desta forma foram classificados provisoriamente como inconclusivos. Além disso, há casos em que a queixa principal não remete a problemas dermatológicos, mas lesões cutâneas são observadas no paciente e descritas na ficha clinica, sendo assim, estes casos foram classificados como outros.

Os animais diagnosticados ou com quadro suspeito de atopia, segundo os critérios de Favrot et al. (2010), tiveram os seus prontuários foto documentados para posterior análise da terapêutica empregada. Apenas os prontuários e as anotações dos Médicos Veterinários foram analisadas, não sendo realizado acompanhamento clínico dos referidos animais.

\section{Resultados e Discussão}

O estudo retrospectivo dos atendimentos realizados no período de janeiro a dezembro/2012 demonstrou que neste intervalo de tempo foram realizados 3375 atendimentos, sendo 2871 de caninos, 495 de felinos, 1 lagomorfo e em 8 fichas não havia a identificação da espécie (Tabela 1). Entre estes animais havia 1802 fêmeas, 1543 machos e 30 fichas não possuíam a identificação do sexo. Das 3375 fichas analisadas, 21,5\% (717/3375) eram referentes a afecções do sistema tegumentar e 78,5\% (2658/3375) estavam relacionados a enfermidades dos sistemas respiratório, gastrointestinal, renal, além de casos do setor oncológico, cirúrgico e emergência. Este percentual de atendimento dermatológico concorda com o que é observado a nível mundial, que oscila entre 21 e $42 \%$ das consultas veterinárias realizadas (Bachir \& Yamani, 2011, Silva et al., 2009). No entanto, difere daquele registrado por Cardoso et al. (2011) no estado do Paraná (Brasil), que observou um percentual de atendimentos dermatológicos de aproximadamente $31 \%$ em um Hospital Veterinário Universitário.

Dentre os animais com afecções no sistema tegumentar foram identificados casos de sarna sarcoptica, sarna demodécica, dermatites alérgicas, dermatites bacterianas e dermatomicoses, confirmando a informação da literatura de que as principais causas de afecções dermatológicas diagnosticadas na rotina clínica incluem doenças parasitárias, alérgicas e hormonais, dando origem a sinais clínicos como prurido, vermelhidão, descamação, alopecia, hiperqueratose e hiperpigmentação. 
Tabela 1. Relação dos animais atendidos catalogados por espécie e por tipo de atendimento no período de janeiro a dezembro/2012 no Hospital Universitário Veterinário, Salvador, Bahia.

\begin{tabular}{lccc}
\hline & Clínica geral & Dermatologia & Total \\
\hline Caninos & 2197 & 674 & 2871 \\
Felinos & 452 & 43 & 495 \\
Coelho & 1 & 0 & 1 \\
Não identificado & 8 & 0 & 8 \\
\hline Total & 2658 & 717 & 3375 \\
\hline
\end{tabular}

Observa-se que dentre todas as dermatologias catalogadas as de origem alérgicas são as que possuem maior frequência, enquanto que as de origem endócrina são as que apresentam um diminuto número de casos, tal resultado reflete a ascensão dos problemas alérgicos, o que pode ser justificado pela maior aquisição de animais de raças predispostas (Gráfico 1).

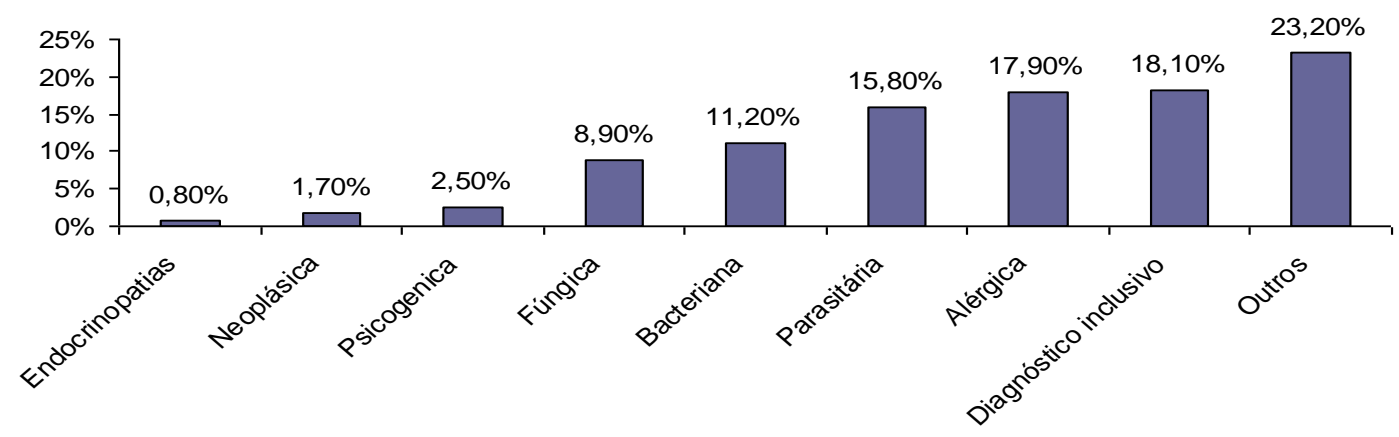

Gráfico 1. Dermatopatias atendidas no Hospital de Medicina Veterinária Professor Renato de Medeiros Neto, da Universidade Federal da Bahia.

A maioria das afecções dermatológicas em cães esteve relacionada a reações de hipersensibilidades como dermatite alérgica à picada de ectoparasitas $(\mathrm{n}=56)$, dermatite atópica canina $(n=31)$, alergia alimentar $(n=5)$ e alergia de contato $(n=30)$ (Gráfico 2). Neste estudo, a atopia foi a segunda afecção alérgica mais frequente nesta instituição, convergindo assim com os resultados encontrados por Cardoso et al. (2011), mas divergindo do resultado encontrado por Souza et al. (2009), onde a DAC foi a dermatopatia de etiologia alérgica mais frequente. No Brasil, no estado do Paraná e do Rio Grande do Sul, dentre as dermatopatias alérgicas atendidas há registro de prevalência de 4,29 a 16,5\% de casos de DAPE, 3,88 a $16,7 \%$ de DAC e 1,6 a $3,2 \%$ de HA (Cardoso et al., 2011, Souza et al., 2009).

Todos os animais com o diagnóstico de DA atendidos no HOSPMEV eram da espécie canina. Isto se justifica pelo fato desta afecção ainda não estar completamente elucidada para os felinos e ainda assim é raro o diagnóstico desta doença na espécie supracitada. Algumas raças são mais predispostas a desenvolverem DAC como Golden retriever $(10,41 \%-13,95 \%)$, Labrador retriever $(6,77 \%$ - 13,48\%), Pastor alemão $(6,04 \%$ $17,18 \%)$, West Highland White Terrier $(6,77 \%$ $1,39 \%)$, Boxer $(3,44 \%-1,7 \%)$, Bichon Frise $(3,26 \%-2 \%)$ e Bulldog Francês $(2,6 \%-0,93 \%)$ (Jaeger et al., 2010). No Brasil, um estudo determinou a prevalência desta enfermidade, que foi identificada primariamente em Poodle (22\%), Maltês (14,6\%), Cães mestiços (9,8\%), Dachshound $(7,3 \%)$, Beagle $(7,3 \%)$, Labrador retriever (7,3\%), ShihTzu (4,9\%) e YorkshireTerrier $(4,9 \%)$. No presente estudo observou-se que dentre os cães atópicos atendidos no HOSPMEV, a raça Poodle foi a que apresentou maior acometimento $(n=14)$ (Gráfico 3). Estes resultados diferem daqueles registrados por Jaeger et al. (2010) que avaliaram a prevalência das raças mais acometidas pela dermatite atópica na 
Alemanha, Austrália e Estados Unidos e registraram maior acometimento em Bichon Frise, Boxer, Bullterrier, Cavalier King Charles Spaniel, Bulldog Francês e Pastor Alemão. No entanto, nossos resultados corroboram os de Rondelli et al. (2012)que avaliaram a frequência de dermatite atópica em cães no Brasil e observaram maior prevalência em cães da raça Poodle, Maltês, SRDs, Dachshound e Labrador.

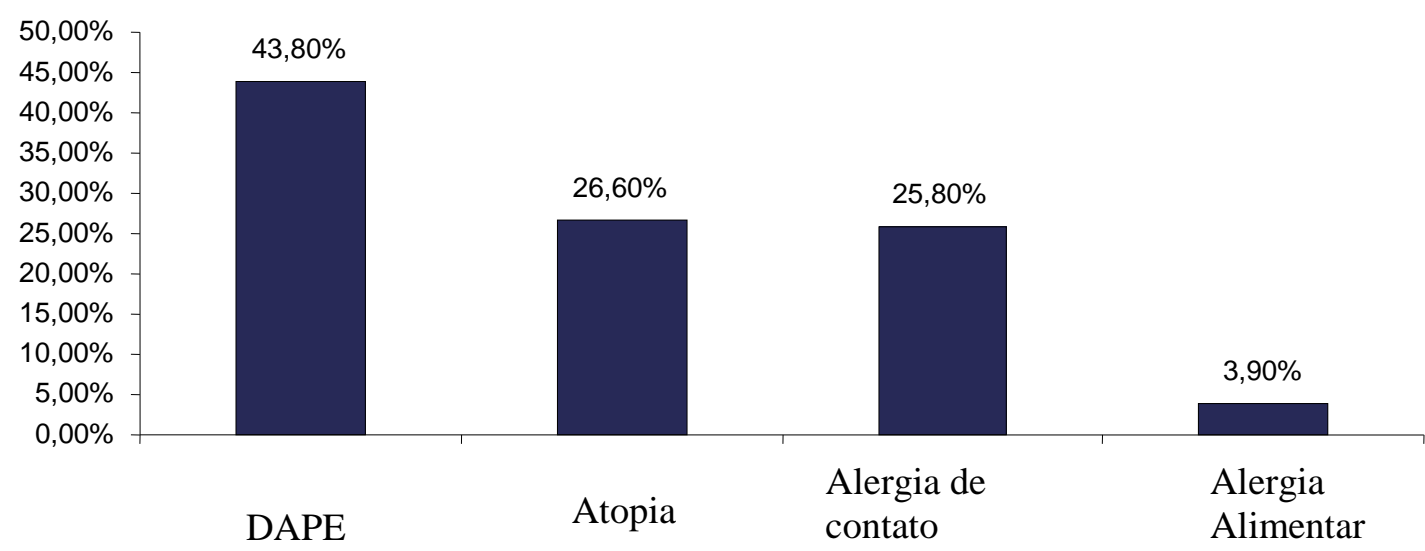

Gráfico 2: Reações de hipersensibilidades encontradas nos animais atendidos no Hospital de Medicina Veterinária Professor Renato de Medeiros Neto, da Universidade Federal da Bahia, na cidade de Salvador, no período de janeiro de 2012 a dezembro de 2012.

No mundo, a idade de aparecimento da dermatite atópica canina variou de 1,5 anos a 1,66 anos, o que demonstra uma maior predileção por animais jovens (Zur et al., 2002, Silva et al., 2009). No Brasil, Rondelli et al. (2012) observa que os diagnósticos de DAC eram realizados com uma média de 6,4 anos, o que corrobora com os resultados obtidos pelo presente estudo no
HOSPMEV ( 6,8 anos), que por sua vez discorda daqueles registrados por Cardoso et al. (2011) que diagnosticaram animais atópicos com idades variando entre 2 e 4 anos. A média de idade obtida pelo presente estudo justifica-se pelo fato de que muitos dos tutores não levam seus animais na fase inicial do processo, ocasionando assim um diagnóstico tardio.

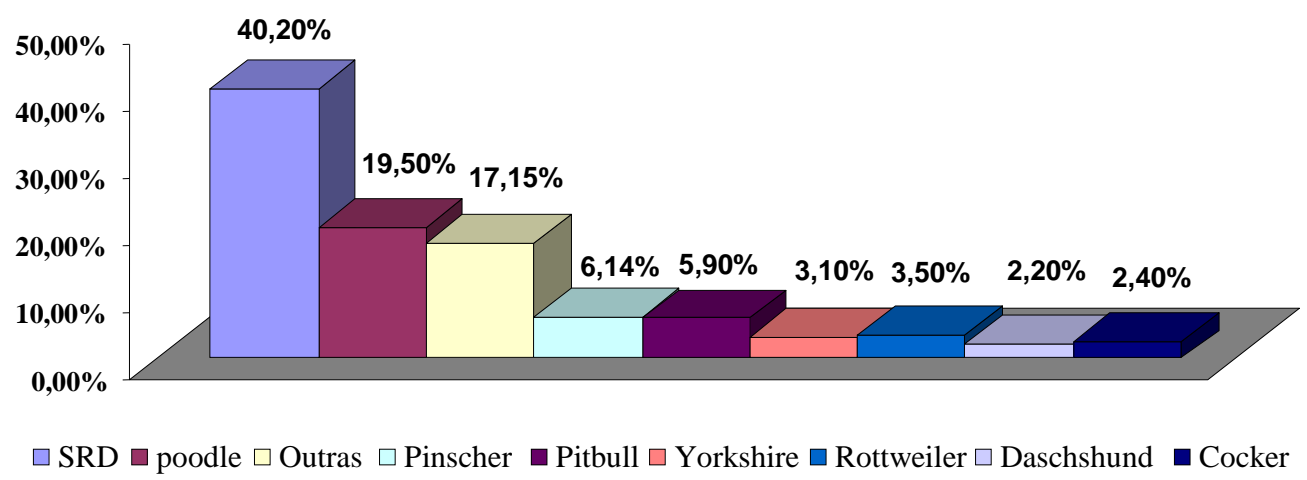

Gráfico 3: Raças de cães com dermatopatias atendidos no Hospital de Medicina Veterinária Professor Renato de Medeiros Neto, da Universidade Federal da Bahia, na cidade de Salvador, no período de janeiro de 2012 a dezembro de 2012.

Zanon et al. (2008) afirmam que há divergências quanto a predisposição sexual desta afecção e que, desta forma, não é possível definir qual a predileção sexual da DAC. Alguns relatos da literatura, como Favrot et al. (2010) relatam uma maior frequência de acometimento em fêmeas do que machos, semelhantes ao registrado no Brasil, onde foi observada uma maior 
frequência de fêmeas atópicas $(75,6 \%)$ do que de machos $(24,4 \%)$ (Rondelli et al., 2012). Quanto à distribuição sexual dos animais atópicos atendidos no hospital veterinário, observou- se uma maior frequência desta enfermidade em fêmeas. Tal fato pode ser justificado como um reflexo dos atendimentos gerais, tendo em vista que nesta instituição atende-se um maior número de fêmeas. É importante destacar que embora os resultados registrados aqui corroborem com alguns estudos e discordem de outros (Favrot et al., 2010), ainda há uma marcada divergência entre os pesquisadores da área com relação à prevalência sexual desta enfermidade (Zanon et al., 2008).

\section{Conclusões}

A dermatologia veterinária é uma das especialidades em maior crescimento na prática clínica e, por vezes, esta é negligenciada por falta de conhecimento das afecções que mais acontecem na região. Ocorrendo assim o subdiagnóstico de algumas enfermidades e a aplicação de protocolos terapêuticos inadequados. Com isso, a partir da frequência das dermatopatias demonstrou-se a importância da dermatologia veterinária na rotina clínica, contribuindo com a identificação das principais afecções dermatológicas que acometem os carnívoros domésticos. Dentre estas, a atopia é considerada a mais importante devido ao seu difícil diagnóstico e ao aumento do número de casos identificados. É de suma importância a existência de estudos em outras regiões, pois desta forma será possível traçar o perfil das dermatopatias no Brasil.

\section{Referências Bibliográficas}

Bachir, P. M. \& Yamani, R. R. (2011). The main skin diseases encontered in pratice canine in the dentraç department of Algeria. Agricultura, Agricultural Practice and Science Journal, 77.

Bianchi, S. P., Correa, R. K. R., Villa-Lobos, W. O. R., Ferreira, R. R. \& Machado, M. L. S. (2008). Atendimentos realizados no ano de 2007 no Serviço de Dermatologia do Hospital de Clínicas Veterinárias da UFRGS. Anais $35^{\circ}$ Conbravet, Gramado, RS, 1, 50.

Cardoso, M. J. L., Machado, L. H. A., Melussi, M., Zamarian, T. P., Carnielli, C. M. \& Júnior Ferreira, J. C. M. (2011). Dermatopatias em cães: revisão de 257 casos. Archives of Veterinary Science, 16, 66-74.

Favrot, C., Steffan, J., Seewald, W. \& Picco, F. (2010). A prospective study on the clinical features of chronic canine atopic dermatitis and its diagnosis. Veterinary Dermatology, 21, 2331.

Jaeger, K., Linek, M., Power, H. T., Bettenay, S. V., Zabel, S., Rosychuk, R. A. W. \& Mueller, R. S. (2010). Breed and site predispositions of dogs with atopic dermatitis: a comparison of five locations in three continents. Veterinary Dermatology, 21, 119-123.

Marsella, R. \& Samuelson, D. (2009). Unravelling the skin barrier: a new paradigm for atopic dermatitis and house dust mites. Veterinary Dermatology, 20, 533-540.

Rondelli, M. C. H., Palacios Junior, R. J. G., Tristao, A. P. P. A., Adams, F. K. \& TinucciCosta, M. (2012). Cães Atópicos: Estudo retrospectivo da resposta clínica a diferentes protocolos terapêuticos. In: Congresso Paulista das Especialidades.

Silva, F. A. N., Carvalho, R. L., Klein, R. P. \& Quessada, A. M. (2009). Posse responsável de cães no bairro Buenos Aires na cidade de Teresina (PI). Ars Veterinaria, 25, 14-17.

Souza, T. M., Fighera, R. A., Schmidt, C., Réquia, A. H., Brum, J. S., Martins, T. B. \& Barros, C. S. L. (2009). Prevalência das dermatopatias não-tumorais em cães do município de Santa Maria, Rio Grande do Sul (2005-2008). Pesquisa Veterinaria Brasileira, 29, 157-162.

Zanon, J. P.; Gomes, L. A.; Cury, G. M. M.; Teles, T.C.; Bicalho,A. P.C. V. (2008). Dermatite Atópica Canina. Semina: Ciências Agrárias, 29, 905-920.

Zur, G., Ihrke, P. J., White, S. D. \& Kass, P. H. (2002). Canine atopic dermatitis: a retrospective study of 266 cases examined at the University of California, Davis, 19921998. Part I. Clinical features and allergy testing results. Veterinary Dermatology, 13, 89-102.

Article History:

Received 4 August 2016

Accepted 8 September 2016

Available on line 21 October 2016

License information: This is an open-access article distributed under the terms of the Creative Commons Attribution License 4.0, which permits unrestricted use, distribution, and reproduction in any medium, provided the original work is properly cited. 